\title{
Review of Application of Internet of Things in Agriculture in India
}

\author{
Priyanka Rathore \\ M. Tech. Scholar \\ Department of Electronics and Communication \\ SIRT, Bhopal
}

\author{
Jyoti Jain, $\mathrm{PhD}$ \\ Head of Department \\ Department of Electronics and Communication \\ SIRT, Bhopal
}

\begin{abstract}
The Internet of Things (IoT), the idea of getting real-world objects connected with each other, will change the way users organize, obtain and consume information radically. Internet of Things (IoT) enables various applications (crop growth monitoring and selection, irrigation decision support, etc.) in Digital Agriculture domain. The Wireless Sensors Network (WSN) is widely used to build decision support systems. These systems overcome many problems in the real-world. One of the most interesting fields having an increasing need of decision support systems is Precision Agriculture (PA). Through sensor networks, agriculture can be connected to the IoT, which allows us to create connections among agronomists, farmers and crops regardless of their geographical differences. With the help of this approach which provides real-time information about the lands and crops that will help farmers make right decisions. The major advantage is implementation of WSN in Precision Agriculture (PA) will optimize the usage of water fertilizers while maximizing the yield of the crops and also will help in analyzing the weather conditions of the field.
\end{abstract}

\section{Keywords}

Internet of Things, Smart Farming, Efficiency, Productivity

\section{INTRODUCTION}

Due to colossal development in advances, cultivating has turned out to be more prevalent and noteworthy. Diverse devices and systems are accessible for advancement of cultivating. As indicated by the UN Food and Agriculture Organization, with a specific end goal to encourage the developing populace of the Earth, the world should deliver $70 \%$ more nourishment in 2050 than it did in 2006[3]. To take care of this demand, ranchers and horticultural organizations are swinging to the Internet of Things for investigation and more noteworthy creation capacities. Web of Things (IoT) can assume enormous part in expanding efficiency, getting colossal worldwide market, thought regarding late patterns of yields. IoT is a system of interconnected gadgets which can exchange information proficiently without human inclusion.

Today numerous rural enterprises swung to embrace IoT innovation for shrewd cultivating to upgrade effectiveness, profitability, worldwide market and different highlights, for example, least human mediation, time and cost and so on. The headway in the innovation guarantees that the sensors are getting littler, complex and more monetary. The systems are likewise effectively available all-inclusive so shrewd cultivating can be accomplished with full promise. Concentrating on empowering development in agribusiness, keen cultivating is the response to the issues that this industry is at present confronting. This should be possible utilizing advanced cells and IoT gadgets. Rancher can get any required information or data also can screen his agrarian division.
The Internet of things (IoT) is the most proficient and critical strategies for advancement of answers for the issues. IoT develop from various building a square which incorporates bunches of sensors, software's; organize parts and other electronic gadgets. Additionally it makes information more powerful. IoT permits to trade the information over the system without human contribution.

In Internet of things, we can speak to things with characteristic way simply like ordinary individual, similar to sensor, similar to auto driver and so forth. This thing is doled out an ip address with the goal that it can exchange information over a system. According to the report created by Garner, toward the finish of 2016 there will be $30 \%$ ascent in consider of associated gadgets contrasted with 2015 . He additionally says that, this tally will increment to 26 billion by 2020 [1]. The IoT innovation is more productive because of following reasons:

1. Global Connectivity through any devices.

2. Minimum human efforts

3. Faster Access

4. Time Efficiency

5. Efficient Communication

The Internet is the worldwide arrangement of interconnected PC organizes that utilization the Internet convention suite (TCP/IP) to connect billions of gadgets around the world. These days more than $46 \%$ of the total populace utilizes the Internet [3]. It has revolutionarily affected culture and business, including the ascent of close moment correspondence by electronic mail, texting and voice over Internet Protocol (VoIP) phone calls, two-way intuitive video calls, interpersonal interaction, and web based shopping destinations. Also, Internet availability turned into the standard for some business applications and is today necessary piece of numerous endeavors, mechanical and customer items to give access to data. In any case, the Internet utilization still fundamentally centers on human connection and checking through applications and interfaces. IoT is the following phase of the Internet in which additionally physical things impart. IoT joins the ideas "Web" and "Thing" and can subsequently semantically be characterized as "an overall system of interconnected protests exceptionally addressable, in view of standard correspondence conventions" [4]. The idea was first presented by the MIT Auto-ID Center to mark the advancement towards an existence where every physical question can be followed by means of the web by labeling them with Radio Frequency Identification (RFID) transponders [5]. Meanwhile, the significance is extended towards an internet of keen associated objects that are setting touchy and can be recognized, detected and controlled remotely by utilizing sensors and actuators [6-8]. In the IoT 
each 'thing' is interestingly identifiable, furnished with sensors and associated continuous to the web. Thus, the Internet will be profoundly implanted in the day by day life of buyers and organizations. Imperceptible innovation works off camera, powerfully reacting to how we need "things" to act. The IoT is relied upon to be the following Internet insurgency. To date, the world has conveyed around 5 billion "brilliant" associated things. Forecasts demonstrate that there will be up to 50 billion associated gadgets by 2020 and in our lifetime we will encounter existence with a trillion-hub arranges [9].

\section{SCOPE OF IOT IN AGRICULTURE}

Agriculture is the principle spine of India's Economic development. The most critical hindrance that emerges in customary cultivating is climatic change. The quantity of impacts of climatic change incorporates overwhelming precipitation, most extraordinary tempest and warmth waves, less precipitation and so forth. Because of these the efficiency reductions to real degree. Climatic change additionally raises the ecological results, for example, regular changes in life cycle of plants. To help the profitability and limit the boundaries in horticulture field, there is have to utilize inventive innovation and procedures called Internet of Things. Today, the Internet of Things (IoT) is changing towards agribusiness industry and empowering ranchers to rival the gigantic difficulties they confront. Ranchers can get immense data and information about ongoing patterns and innovation utilizing IoT.

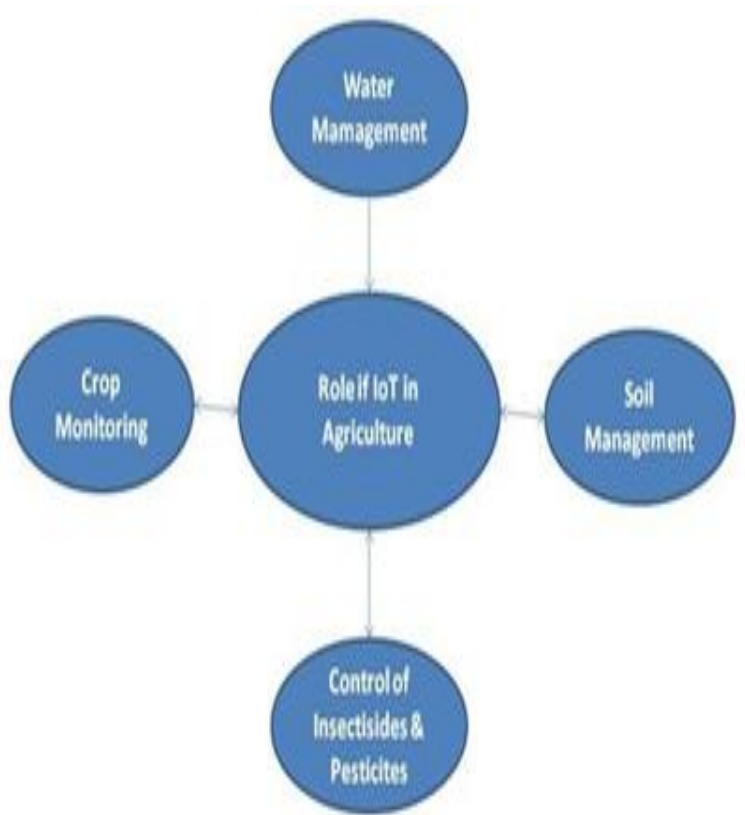

Figure 1: Role of IoT in Agriculture

The smart farming business sector is relied upon to reach $\$ 18.45$ Billion of every 2022 , at a CAGR of $13.8 \%$. BI gauges that 75 million IoT gadgets will be delivered for horticultural uses in 2020, at a CAGR of $20 \%$. IoT gadgets can be of incredible help in improving the generation and yield in the agribusiness part since these gadgets can be utilized to screen soil corrosiveness level, temperature, and different factors. In addition, savvy agribusiness will help in observing domesticated animals efficiency and wellbeing also. IoT sensors are fit for furnishing ranchers with data about harvest yields; precipitation, bother pervasion, and soil nourishment are important to generation and offer exact information which can be utilized to enhance cultivating methods after some time. Web of things, with its continuous, precise and shared qualities, will convey extraordinary changes to the farming inventory network and give a basic innovation to building up a smooth stream of horticultural coordination's [4].

The key advantages of using IoT in enhancing farming are as follows:

1. Water administration can be effectively done utilizing IoT with no wastage of water utilizing sensors.

2. IoT serves to consistent screen the land with the goal that safety measures can be taken at beginning time.

3. It expands profitability, diminish manual work, lessen time and makes cultivating more productive.

4. Crop checking can be effectively done to watch the development of product.

5. Soil administration, for example, $\mathrm{PH}$ level, Moisture content and so forth can be distinguished effectively with the goal that agriculturist can sow seeds as indicated by soil level.

6. Sensors and RFID chips helps to perceive the maladies happened in plants and products. RFID labels send the EPC (data) to the peruser and are shared over the web. The agriculturist or researcher can get to this data from a remote place and take vital activities; automatically products can be shielded from coming infections [2].

7. Crop deals will be expanded in worldwide market. Rancher can undoubtedly associate with the worldwide market without limitation of any land territory.

\section{LITERATURE REVIEW OF IOT IN AGRICULTURE}

The idea of the Internet of Things initially wound up well known in 1999, through the Auto-ID Center at MIT and related market-examination distributions. Scientists have proposed diverse models for horticulture segment with one or various advances said above e.g. water system framework in light of soil water estimation to choose water system measure of the water is depicted in [1]. Which utilizes the Bluetooth show for the correspondence which has its own confinements like restricted range and gadget settlement? In the time of 2009, a creator recommended planning for the power supplies to the sensors which will help in enhance vitality proficiency [2]. Utilization of IoT in agribusiness is said by a creator in paper [3]. Anyway it demonstrates absence of interoperability which is fundamental when we discuss expansive horticultural fields. For correlation of vitality utilization between two apparatuses, Jinsoo han has given an approach in paper [4] distributed in 2011. N.K. Suryadevara, S.C. Mukhopadhyay has utilized ideas of inescapable processing, information accumulation and so on to screen the ecological components utilizing Zigbee [5] in their paper. Increment in number of sensors is proposed by the creator to enhance the exactness of the information gathered. Anyway it may raise the issue of more power utilization as more hubs has been conveyed. Way to deal with give the constant data to the agriculturists about the land and harvests is characterized in the paper [7], which gives the essential data yet it's an independent framework. In the time of 2015 ideas of IoT, distributed computing, Mobile registering are utilized as a part of keen horticulture in paper [8], where by Prem Prakash Jayaraman, Doug Palmer, Arkady 
Zaslavsky the idea of phenonet was presented [9], which is system of shrewd remote sensor hubs who imparts the data to each different and in addition focal framework. However both the papers anyway do not give any understanding of the information despite the fact that substantial measure of helpful information is produced. In spite of the fact that analysts have proposed few models in horticulture area utilizing at least one of the innovations said, we mean to build up a coordinated arrangement of various functionalities with information elucidation and more straightforward interface.

Phenonet [5] is a horticultural phenotyping field research center, including an assortment of product considers that are being directed utilizing cutting edge IoT advances [6], including sensor systems, IP cameras, versatile cell phones, and related information examination. These empower close continuous catch of product information for evaluating and anticipating crop execution (both here and now and long haul) for any given natural, soil, preparation, and water system conditions, including climate conditions, plant shade temperature, soil dampness, soil quality and saltiness, compost use, and water system. Phenonet is helping plant researcher and cultivators accomplish the accompanying: (1) distinguish the impact of various conditions on an assortment of harvests in certifiable open air cultivate situations; (2) comprehend water asset utilization keeping in mind the end goal to oversee it successfully; (3) think about the effect of different manures; (4) get ongoing information to figure trim execution; and (5) share information and results. To better clarify Phenonet, we give extra insights about example considers led in Phenonet. One of these examinations plan to assess the impact of sheep touching on trim re-development by taking a gander at root action, water utilize, rate of harvest development, and product yield. In this examination, soil dampness sensors sent at numerous profundities and shade temperature sensors are utilized to track the yield roots' extraction of water from the dirt all through the developing season. This data is then used to gauge and evaluate root action and product development. Figure 1 exhibits an outline of this Phenonet think about that is directed on a square of land that is separated into several plots. A plot is a convergence of a line and a segment in a square of land and has a particular treatment to the product being contemplated. For instance, each plot may have an alternate use of composts or host the utilization of similar manure at various circumstances. This Phenonet consider requires bolster for continuous gathering and conveyance of information to scholars, who at that point share their bits of knowledge with producers and ranchers, and related logical associations. A portion of the fundamental difficulties in supporting such investigations and furthermore typical cultivating exercises incorporate the accompanying:

- Capturing the expansive volume of heterogeneous information delivered by an assortment of IoT sensors (and potentially manual estimations), and doing this for a huge number and assortment of exercises including distinctive investigations and additionally trims.

- Supporting the incorporation and utilization of any IoT gadget, including all economically accessible sensors, camera, climate stations, and so on, as this will accomplish a bring-your-own particular sensor model of activities that will permit agriculturists, producers, and researchers to exploit less expensive/more able IoT sensors, and additionally singular inclinations and spending plans.
- Integrating heterogeneous information from such an awesome assortment of IoT gadgets and furthermore recorded yield execution information delivered by past examinations (such information and results are commonly accessible in CSV documents that make it harder to utilize, investigate, investigate, and share).

- Providing crop execution information investigation programming and related apparatuses for do-itwithout anyone else's help inquiry, examination and perception of gathered information over numerous investigations.

\section{CONCLUSION}

Farming will assume imperative part in next couple of years in nation. Subsequently there is need of keen cultivating. Web of Things will upgrade brilliant cultivating. IoT works in various areas of cultivating to enhance time productivity, water administration, trim observing, soil administration, control of bug sprays and pesticides and so on. It likewise limits human endeavors, disentangles strategies of cultivating and increases shrewd cultivating. Alongside these highlights brilliant cultivating can develop the market for rancher with single touch and least endeavors.

\section{REFERENCES}

[1] Jim Chase: The Evolution of the Internet of Things. White Paper, Texas Instruments, September, 2013.

[2] Deeksha Jain, P. Venkata Krishna and V. Saritha, "A Study on Internet of Things based Applications", 2012.

[3] http://www.businessinsider.com/internet-of-things-smartagriculture-2016-10?IR=T

[4] Xiaohui Wang and Nannan Liu, "The application of internet of things in agricultural means of production supply chain management", Journal of Chemical and Pharmaceutical Research, 2014, 6(7):2304-2310, ISSN: 0975- 7384,2014.

[5] Schoenberger, C.R., The internet of things. Forbes, 2002(3/18).

[6] Atzori, L., A. Iera, and G. Morabito, The Internet of Things: A survey. Computer Networks, 2010. 54(15): p. 2787-2805.

[7] Kortuem, G., et al., Smart objects as building blocks for the Internet of things IEEE Internet Computing, 2010. 14(1): p. $44-51$.

[8] Porter, M.E. and J.E. Heppelmann, How Smart Connected Objects Are Transforming Competition. Harvard Business Review, 2014(November): p. 65-88.

[9] Castaneda, C. Internet of Things to Become Cornerstone of Excellent Customer Service, Finds Frost \& Sullivan. 2015 [cited 2016 June 29th];

[10] Sean Dieter Tebje Kelly, Nagender Kumar Suryadevara, and Subhas Chandra Mukho padhyay, Fellow, IEEE, "Towards the Implementation of IoT for Environmental Condition Monitoring in Homes", IEEE Sensors Journal, Vol-13, No.10, October 2013.

[11] Mihai T Lazarescu,"Dsignon of a WSN platform for long term envirnomental monitoring for IoT applications", IEEE journal, On emerging and selected topics in circuits and systems, Vol.3, no.1, march2013. 
[12] Mohmad Rawidean Mohd Kassim, Ibrahim Mat, Ahmad Nizar Harun, "Wireless Sensor Network in Precision Agriculture Application", 978-1-4799-4383-8/14 -2014 IEEE.

[13] Hemlata Channe, Sukhesh Kothari, Dipali kadam,"Multidisciplinary model for Smart Agriculture using IoT, Sensors, Cloud-Computing, Mobile
Computing \& Big data Analysis", ISSN: 2229-6093.

[14] Prem Prakash Jayaraman,Doug Palmer, Arkady Zaslavsky, Dimitrios Georgakopoulos, "Do-it-Yourself Digital Agriculture Applications with Semantically Enhanced IoT Platform",2015 IEEE Tenth International Conference (ISSNIP) Singapore,7-9 April 2015. 